\title{
New frontiers in the neuroscience of the sense of agency
}

\section{Nicole David*}

Department of Neurophysiology and Pathophysiology, University Medical Center Hamburg-Eppendorf, Hamburg, Germany

\section{Edited by:}

Sven Bestmann, University College

London, UK

Reviewed by:

Manos Tsakiris, University of

London, UK

Sukhvinder Obhi, Wilfrid Laurier

University, Canada

*Correspondence:

Nicole David, Department of Neurophysiology and

Pathophysiology, University Medical Center Hamburg-Eppendorf,

Martinistr. 52, 20246 Hamburg,

Germany.

e-mail:ndavid@uke.de
The sense that I am the author of my own actions, including the ability to distinguish my own from other people's actions, is a fundamental building block of our sense of self, on the one hand, and successful social interactions, on the other. Using cognitive neuroscience techniques, researchers have attempted to elucidate the functional basis of this intriguing phenomenon, also trying to explain pathological abnormalities of action awareness in certain psychiatric and neurological disturbances. Recent conceptual, technological, and methodological advances suggest several interesting and necessary new leads for future research on the neuroscience of agency. Here I will describe new frontiers for the field such as the need for novel and multifactorial paradigms, anatomically plausible network models for the sense of agency, investigations of the temporal dynamics during agentic processing and ecologically valid virtual reality (VR) applications.

Keywords: sense of agency, connectivity, temporal dynamics, virtual reality

\section{INTRODUCTION}

The cognitive neuroscience approach to the sense of agency-that is, the sense that it is me or someone else causing or controlling a movement or change in the outside world (e.g., Gallagher, 2000) — views it as an operationalizable construct that can be broken down into paradigms amenable to neuroscience techniques (Christoff et al., 2011), such as functional magnetic resonance imaging (fMRI), electro- or magnetoencephalography (EEG or MEG), and transcranial magnetic stimulation (TMS). Yet, from the late 1990s to date, the sense of agency received only little attention in neuroscience research, despite its relevance to a variety of psychiatric and neurological syndromes (Blakemore et al., 2002) and its key role for self-consciousness and social interaction (Gallagher, 2000; De Jaegher and Froese, 2009). Compared with related areas of interest in cognitive neuroscience, such as the mirror neuron system or theory of mind, the number of studies in cognitive neuroscience which addressed the sense of agency can be considered small. Sperduti and colleagues (2011) in a recent meta-analysis identified only 31 neuroimaging studies directly related to the sense of agency and published since 1998. Half of these studies addressed only one of many different aspects of agency processing in the brain, namely, the identification of brain regions that respond to an intact versus disrupted sense of self-agency by introducing sensorimotor congruence versus incongruence (Sperduti et al., 2011). However, advancing technologies, such as in virtual reality (VR) and robot systems, developments in neuroscience methodologies, such as analyses of connectivity or interactions between brain regions, as well as conceptual developments of the sense of agency have opened up new, intriguing doors for the neuroscience of agency. In the following, I will introduce some recent developments in the field, current outstanding questions, and consequent directions for future research.

\section{MULTIFACTORIAL SENSE OF AGENCY: THE PERFECT EXPERIMENT}

An example of the various stages and processes involved in agentic processing is writing this article. It started with my intention to write this article because I considered the topic important. Now, while I am writing, efference-based motor control signals make me hit certain keys on my keyboard. At the same time, self-monitoring processes and the visual feedback from my screen prevent me from making typos and help to correct myself when a wrong key has been hit or when a key remained unresponsive to my key press. Some of these processes may happen automatically without me reflecting upon them or being able to control them voluntarily. Yet, I implicitly experience myself as the agent who is thinking these words and putting them onto paper, I might become aware of the unresponsive key, and eventually I will explicitly experience myself as the author of this article as it gets published. This example highlights that the sense of agency is complex and dynamic, multilayered, and multifactorial (Wegner and Sparrow, 2004; Pacherie, 2008; Synofzik et al., 2008; Gallagher, 2012). Nonetheless, different authors have put different emphasis on single aspects, strictly conceiving the sense of agency in very narrow ways. Some authors have proposed motor efference signals and motor intention, such as during active versus passive movements or temporal binding of action and action outcome during voluntary versus non-voluntary movements, as primary constituents for the sense of agency (Haggard, 2005; Tsakiris et al., 2005; Moore and Obhi, 2012), some have promoted a key role of proprioceptive signals (Balslev et al., 2007), others have explained the sense of agency rather cognitively (Aarts et al., 2005; Metcalfe and Greene, 2007), etc. The two predominant experimental approaches have addressed (1) the "intentional binding" effect, i.e., the subjective compression of the time between a voluntary action and its experienced sensory outcome (Haggard 
et al., 2002; Moore and Obhi, 2012) and (2) the "comparator model," which posits that the sense of agency results from a sensorimotor matching between a predicted and the actual state (for a comprehensive overview and discussion see Synofzik et al., 2008). As a consequence, neuroscientific operationalizations of agency have been diverse (David et al., 2008), with the employment of a sensorimotor mismatch being the most common (see Sperduti et al., 2011). In contrast, and unfortunately, we hardly know anything about the brain basis of intentional binding (Moore and Obhi, 2012), with the exception of Moore et al. (2010) who showed that interruption of the pre-supplementary motor area (pre-SMA) via TMS interfered with intentional binding.

However, there is now reason to believe that the sense of agency is "the product of several contributory elements" (Gallagher, 2012), cues (Wegner and Sparrow, 2004; Moore et al., 2009) or processes (Christoff et al., 2011). Synofzik and colleagues (2008; Vosgerau and Synofzik, 2012; also Moore and Fletcher, 2012) proposed a multifactorial weighting model, in which internal cues from efferent, reafferent, intention, and environment interact dynamically, following principles of optimal cue integration given a situation. Thus, a more topical neuroscience experiment would consider many different agency cues at the same time, going beyond the mere interruption of the sense of agency by sensorimotor incongruence. Tsakiris and coworkers (2010) made an attempt toward a "multifactorial experiment." They let their participants perform active finger movements or passively lifted the participants' fingers, while providing synchronous or delayed visual feedback of these finger movements via video. The authors (2010) found the biggest self-agency experience when active movements were performed under synchronous feedback. This condition was associated with increased activity in the medial aspect of the premotor cortex (i.e., the SMA). In contrast, the inferior parietal lobule showed higher activation for asynchronous visual feedback, particularly when the movement was passively induced. Similarly, Desmurget and colleagues (2009) dissociated premotor and inferior parietal cortex in patients undergoing invasive electrical stimulation. Experiments like these help to elucidate what a given "neural correlate of the sense of agency" truly reflects, while also allowing for predictions with respect to network activity.

\section{NEURAL NETWORKS AND NETWORK INTERACTIONS UNDERLYING THE SENSE OF AGENCY}

The previous section highlighted the need of a full-fledged account of the sense of agency, capable of integrating the presently available neuroscientific evidence. Despite the fact that highly distributed networks underlie most cognitive processes, an explicit account of how the "neural correlates of agency" interact during agentic processing is missing to date. Only two out of the more than 30 identified neuroimaging studies of agency (Sperduti et al., 2011) investigated network activity by measuring "functional connectivity" (David et al., 2007; Nahab et al., 2011). Functional connectivity seeks to identify highly connected brain regions based on time course correlations of their activity (Sporns et al., 2004). The author and her coworkers (David et al., 2007), for instance, revealed correlated activity of the insula and somatosensory cortex for visual feedback correctly experienced as self-generated, on the one hand, and between the premotor cortex, cerebellum, and posterior parietal cortex for visual feedback correctly experienced as experimenter-generated, on the other hand. Nahab and colleagues (2011) identified three different networks that were active when participants lost control of moving a virtual hand on the screen. Nahab and colleagues (2011) referred to these regions as a mismatch detection network, which was characterized by fast and high-amplitude hemodynamic responses (e.g., in the insula and inferior parietal lobule), and a slower, low-amplitude higher-order processing network (e.g., the prefrontal and posterior parietal cortices). These two networks appeared to be relayed in a third category of brain regions (e.g., cerebellum and basal ganglia).

What remained unclear from Nahab and colleagues' (2011) elegant study was whether and how the three functionally different networks feed into and back to each other. Functional connectivity approaches do not allow for such directional (or causal) assumptions about the influence one network might exert over another (this type of connectivity is termed "effective"; Sporns et al., 2004). Effective connectivity has been successfully assessed for processes related to the sense of agency such as grasping (Grol et al., 2007) and consciousness (Boly et al., 2011) but not for agentic processing. For example, Grol and coworkers revealed that depending on on-line control requirements of a movement, different cortical networks are activated: e.g., grasping large objects increased effective connectivity in a dorsomedial network, grasping small objects in a dorsolateral network. Although neurocomputational models of the sense of agency-similar to those developed for the mirror neuron system or sensorimotor control (e.g., Franklin and Wolpert, 2011)_are lacking to date, an anatomically plausible model of cortical regions interacting during agency situations could be initiated based on what we know from related disciplines or comparative (e.g., anatomical connectivity studies in the monkey) and neuropsychological data. For example, Desmurget and coworkers impressively demonstrated that stimulation of the inferior parietal cortex in awake patients undergoing brain surgery triggered movement intentions or an increased (illusory) awareness that a movement has been performed, while stimulation of premotor regions led to the actual execution of movements without the patients' awareness. Such findings should be incorporated into a neuroanatomical model of the sense of agency, which could then be subsequently tested by "dynamic causal modeling" (DCM). DCM represents a computational approach, which estimates directed influences between variables within a Bayesian framework (Stephan and Friston, 2010) - a promising avenue in light of recent suggestions that the sense of agency may likewise be based on Bayesian principles (Moore and Fletcher, 2012).

\section{BEYOND LOCI: TEMPORAL ASPECTS OF AGENTIC PROCESSING}

Connectivity can also be assessed with other neuroscience techniques such as EEG or MEG, which allow for frequency-resolved analyses of correlations between brain regions or networks (Siegel et al., 2012), for example, by the computation of phase synchronization and spectral coherence, power-power correlations, spectral granger causality, or cross-frequency couplings 
(Cohen et al., 2011). Due to their excellent temporal resolution, EEG and MEG allow us to answer questions about the timing aspects of various processes underlying the sense of agency. Answering these questions becomes also relevant for two recentand related-discussions about: (1) possible indices and the relationship of pre-reflective versus reflective level of action awareness (Gallagher, 2000, 2012; Synofzik et al., 2008); (2) whether the sense of agency is primarily based on sensorimotor prediction versus cognitive reconstruction/inference (Haggard, 2005; Moore and Obhi, 2012).

With respect to these discussions, Gentsch and SchützBosbach (2011; see also Kühn et al., 2011) recently observed a reduced $\mathrm{N} 1$ component, that is, a negative deflection at $100 \mathrm{~ms}$ post-stimulus onset, for a visual stimulus that was self- as opposed to computer-generated. This was taken as the EEG correlate of sensorimotor attenuation, a predictive mechanism for the expected sensory consequences of one's own actions (Blakemore et al., 1998), and an indicator of the pre-reflective level of agency - as this effect is not necessarily associated with correct agency judgments (Kühn et al., 2011). Instead, a later positive deflection in the EEG (i.e., the so-called P3a component peaking at $250-280 \mathrm{~ms}$ ) was increased on trials that were afterwards (i.e., long after $300 \mathrm{~ms}$ ) evaluated as self-generated (Kühn et al., 2011). These results not only provided evidence for potential EEG correlates of pre-reflective versus reflective agency, but also suggested that both levels of the sense of agency are better characterized by early predictive processes. However, findings showing (1) a dissociation between subjective movement awareness/intention and movement execution (Desmurget et al., 2009); (2) that we can also feel causally effective even for events out of our control (Wegner and Wheatley, 1999) and (3) that we have a general bias to over-attribute external events to ourselves (van den Bos and Jeannerod, 2002) suggest the opposite. Moreover, Obhi and colleagues (Strother et al., 2010; Obhi and Hall, 2011) provided evidence for a dissociation between pre-reflective and reflective agency, as assessed by the intentional binding effect and selfreported judgments about agency in a joint-action setting (i.e., several actors), suggesting that both levels of the sense of agency are supported by different processes.

Thus, a reconciliatory view, emerging from recent behavioral data, posits that both prediction and reconstruction constitute the sense of agency but that they are differently weighted for some situations compared to others: e.g., the role of reconstructive processes increases when sensorimotor signals are weak (Moore et al., 2009; Voss et al., 2010; Gentsch et al., 2012; Moore and Obhi, 2012). Identifying the functional basis of a reflective versus prereflective level of agency represents a necessary step for future research in the field.

To date, only a few authors explicitly approached the sense of agency with EEG (Gentsch and Schütz-Bosbach, 2011; Kühn et al., 2011; Gentsch et al., 2012), exclusively focusing on eventrelated potentials. This classical approach, however, can tell us only little about temporal brain dynamics occurring during agentic processing; rather, it represents a static snapshot of the brain's activity at the scalp level (Makeig et al., 2004). Future EEG (or MEG) experiments addressing the sense of agency may want to profit from recent methodological/technological advances, which suggest novel and more sophisticated analyses. These are capable of modeling ongoing brain dynamics, such as timefrequency representations of underlying field potentials (i.e., at the source level), while also allowing researchers to measure dynamic interactions between the nodes of a possible agency network (Makeig et al., 2004; Siegel et al., 2012). This appears to be a more appropriate approach, given the recent conceptual developments of the sense of agency as a dynamic multifactorial weighting process.

\section{ECOLOGICAL VALIDITY}

Critical voices rightfully questioned the ecological validity of many agency paradigms for several reasons. First, most paradigms that manipulated sensorimotor contingencies, such as cursor movements deviating from minimal joystick movements (Farrer and Frith, 2002; David et al., 2007) or sounds/shapes deviating from associated button presses (Spengler et al., 2009; Kühn et al., 2011), investigated effects on disembodied or extracorporeal events as operationalization of an embodied phenomenon. Second, participants might process such paradigms differently, both phenomenologically and neurally, compared to when they can see/feel their own hands and bodies. Third, many operationalizations of agency "imposed binary outcomes to what is likely a complex and dynamic cognitive process" (Nahab et al., 2011).

As a neuroscientist investigating the sense of agency, I am aware of the limitations cognitive neuroscience techniques impose on the idea of increased ecological validity. First, participants are restricted in the movements they can make in magnetic resonance imaging (MRI) and encephalographic (EEG) environments, to prevent causing artifacts in the recording of neural data. Second, increasing ecological validity goes in hand with increasing complexity and the question what of a given "neural correlate" really reflects (compare to Yomogida et al., 2010). However, recent work suggested several ways to trading off such limitations and ecological validity (e.g., David et al., 2006, 2007). VR techniques, in particular, provide a promising avenue, also for simulating and rehabilitating disorders of action monitoring/awareness (Ionta et al., 2011; Verschure, 2011). In several studies, Blanke and his coworkers demonstrated the application of VR along with fMRI or EEG (Ionta et al., 2011; Lenggenhager et al., 2011). For example, Lenggenhager and colleagues (2011) used VR technology and visuotactile conflict to manipulate self-localization. Wearing an EEG cap and optical markers attached to their bodies, participants were placed within a VR tracking arena. The signals provided by these markers were fed into a real-time animated, life-sized virtual character, which appeared on a large projection screen in front of them. Employing a variation of the rubber-hand illusion paradigm (Lenggenhager et al., 2007), the authors then recorded EEG during the synchronous or asynchronous stroking of the participants and the avatar's back. Although these studies (Ionta et al., 2011; Lenggenhager et al., 2011) rather address the sense of body ownership, similar scenarios could be developed to address the sense of agency by contrasting conditions of, for example, present vs. lacking motor intentions, matching vs. mismatching efferent, and reafferent signals, etc., while measuring the degree of agentic experience in the VR. 


\section{CONCLUDING REMARKS}

The sense of agency represents an intriguing phenomenon, amenable to cognitive neuroscience techniques despite its complexity. Although we have just started to elucidate the functional anatomy of the sense of agency, "methodological stagnation" already characterizes cognitive neuroscience approaches to the sense of agency. Recent conceptual developments, on the one hand, and general technological or methodological developments, on the other hand, allow for novel experimental paradigms and analyses, which could - in turn-feed back into further

\section{REFERENCES}

Aarts, H., Custers, R., and Wegner, D. M. (2005). On the inference of personal authorship: enhancing experienced agency by priming effect information. Conscious. Cogn. 14, 439-458.

Balslev, D., Cole, J., and Miall, R. C. (2007). Proprioception contributes to the sense of agency during visual observation of hand movements: evidence from temporal judgments of action. J. Cogn. Neurosci. 19, 1535-1541.

Blakemore, S. J., Wolpert, D. M., and Frith, C. D. (1998). Central cancellation of self-produced tickle sensation. Nat. Neurosci. 1, 635-640.

Blakemore, S. J., Wolpert, D. M., and Frith, C. D. (2002). Abnormalities in the awareness of action. Trends Cogn. Sci. 6, 237-242.

Boly, M., Garrido, M. I., Gosseries, O., Bruno, M. A., Boveroux, P., Schnakers, C., Massimini, M., Litvak, V., Laureys, S., and Friston, K. (2011). Preserved feedforward but impaired top-down processes in the vegetative state. Science 332, $858-862$.

Christoff, K., Cosmelli, D., Legrand, D., and Thompson, E. (2011). Specifying the self for cognitive neuroscience. Trends Cogn. Sci. 15, 104-112.

Cohen, M. X., Wilmes, K., and van de Vijver, I. (2011). Cortical electrophysiological network dynamics of feedback learning. Trends Cogn. Sci. $15,558-566$

David, N., Bewernick, B. H., Cohen, M. X., Newen, A., Lux, S., Fink, G. R., Shah, N. J., and Vogeley, K. (2006). Neural representations of self versus other: visual-spatial perspective taking and agency in a virtual balltossing game. J. Cogn. Neurosci. 18, 898-910.

David, N., Cohen, M. X., Newen, A., Bewernick, B. H., Shah, N. J., Fink, G. R., and Vogeley, K. (2007). The extrastriate cortex distinguishes between the consequences of one's own and others' behavior. Neuroimage 36, 1004-1014.
David, N., Newen, A., and Vogeley, K. (2008). The "sense of agency" and its underlying cognitive and neural mechanisms. Conscious. Cogn. 17, 523-534.

De Jaegher, H., and Froese, T. (2009). On the role of social interaction in individual agency. Adapt. Behav. 17, 444-460.

Desmurget, M., Reilly, K. T., Richard, N., Szathmari, A., Mottolese, C., and Sirigu, A. (2009). Movement intention after parietal cortex stim811-813.

Farrer, C., and Frith, C. D. (2002). Experiencing oneself vs another person as being the cause of an action: the neural correlates of the experience of agency. Neuroimage 15, 596-603.

Franklin, D. W., and Wolpert, D. M. (2011). Computational mechanisms of sensorimotor control. Neuron 72 425-442.

Gallagher, S. (2000). Philosophical conceptions of the self: implications for cognitive science. Trends Cogn. Sci. 4, 14-21.

Gallagher, S. (2012). Multiple aspects in the sense of agency. New Ideas Psychol. 30, 15-31.

Gentsch, A., Kathmann, N., and Schütz-Bosbach, S. (2012). Reliability of sensory predictions determines the experience of self-agency. Behav. Brain Res. 228, 415-422.

Gentsch, A., and Schütz-Bosbach, S. (2011). I did it: unconscious expectation of sensory consequences modulates the experience of self-agency and its functional signature. J. Cogn. Neurosci. 23, 3817-3828.

Grol, M. J., Majdandzić, J., Stephan, K. E., Verhagen, L., Dijkerman, H. K., Bekkering, H., Verstraten, F. A. J., and Toni, I. (2007). Parietofrontal connectivity during visually guided grasping. J. Neurosci. 27, 11877-11887.

Haggard, P. (2005). Conscious intention and motor cognition. Trends Cogn. Sci. 9, 290-295. ulation in humans. Science 324,

conceptual developments of the sense of agency. Thus, the neuroscience of the sense of agency remains topical, offering several interesting, evident, and necessary leads for further exploration.

\section{ACKNOWLEDGMENTS}

This work was supported by the Deutsche Forschungsgemeinschaft (DA 1358/1-1) and the European Union ("eSMCs", FP7ICT-2009-6 No. 270212). The author would like to thank Brittany S. Holmes for comments on a previous version of the manuscript.

Haggard, P., Clark, S., and Kalogeras, J. (2002). Voluntary action and conscious awareness. Nat. Neurosci. 5, 382-385.

Ionta, S., Gassert, R., and Blanke, O. (2011). Multi-sensory and sensorimotor foundation of bodily selfconsciousness-an interdisciplinary approach. Front. Psychol. 2:383. doi 10.3389/fpsyg.2011.00383

Ionta, S., Heydrich, L., Lenggenhager, B., Mouthon, M., Fornari, E. Chapuis, D., Gassert, R., and Blanke, O. (2011). Multisensory mechanisms in temporo-parietal cortex support self-location and first-person perspective. Neuron 70 , 363-374.

Kühn, S., Nenchev, I., Haggard, P. Brass, M., Gallinat, J., and Voss, M. (2011). Whodunnit? electrophysiological correlates of agency judgements. PLoS ONE 6:e28657. doi 10.1371/journal.pone.0028657

Lenggenhager, B., Halje, P., and Blanke, O. (2011). Alpha band oscillations correlate with illusory self-location induced by virtual reality. Eur. J. Neurosci. 33 , 1935-1943.

Lenggenhager, B., Tadi, T., Metzinger, T., and Blanke, O. (2007). Video ergo sum: manipulating bodily self-consciousness. Science 317 1096-1099.

Makeig, S., Debener, S., Onton, J., and Delorme, A. (2004). Mining eventrelated brain dynamics. Trends Cogn. Sci. 8, 204-210.

Metcalfe, J., and Greene, M. J. (2007) Metacognition of agency. J. Exp. Psychol. 136, 184-199.

Moore, J. W., and Fletcher, P. C. (2012) Sense of agency in health and disease: a review of cue integration approaches. Conscious. Cogn. 21, 59-68.

Moore, J. W., and Obhi, S. S. (2012). Intentional binding and the sense of agency: a review. Conscious. Cogn. 21, 546-561.

Moore, J. W., Ruge, D., Wenke, D. Rothwell, J., and Haggard, P. (2010). Disrupting the experience of control in the human brain: pre-supplementary motor area contributes to the sense of agency. Proc. R. Soc. B Biol. Sci. 277, 2503-2509.

Moore, J. W., Wegner, D. M., and Haggard, P. (2009). Modulating the sense of agency with external cues. Conscious. Cogn. 18, 1056-1064.

Nahab, F. B., Kundu, P., Gallea, C. Kakareka, J., Pursley, R., Pohida, T., Miletta, N., Friedman, J., and Hallett, M. (2011). The neural processes underlying self-agency. Cereb. Cortex 21, 48-55.

Obhi, S. S., and Hall, P. (2011). Sense of agency and intentional binding in joint action. Exp. Brain Res. 211, 655-662.

Pacherie, E. (2008). The phenomenology of action: a conceptual framework. Cognition 107, 179-217.

Siegel, M., Donner, T. H., and Engel, A. K. (2012). Spectral fingerprints of large-scale neuronal interactions. Nat. Rev. Neurosci. 13, 121-134.

Spengler, S., von Cramon, D. Y., and Brass, M. (2009). Was it me or was it you? How the sense of agency originates from ideomotor learning revealed by fMRI. Neuroimage 46, 290-298.

Sperduti, M., Delaveau, P., Fossati, P., and Nadel, J. (2011). Different brain structures related to selfand external-agency attribution: a brief review and metaanalysis. Brain Struct. Funct. 216, 151-157.

Sporns, O., Chialvo, D. R., Kaiser, M., and Hilgetag, C. C. (2004). Organization, development and function of complex brain networks. Trends Cogn. Sci. 8, 418-425.

Stephan, K. E., and Friston, K. J. (2010). Analyzing effective connectivity with fMRI. Wiley Interdiscip. Rev. Cogn. Sci. 1, 446-459.

Strother, L., House, K. A., and Obhi, S. S. (2010). Subjective agency and awareness of shared actions. Conscious. Cogn. 19, 12-20.

Synofzik, M., Vosgerau, G., and Newen, A. (2008). Beyond the comparator 
model: a multifactorial two-step account of agency. Conscious. Cogn. 17, 219-239.

Tsakiris, M., Haggard, P., Franck, N., Mainy, N., and Sirigu, A. (2005). A specific role for efferent information in self-recognition. Cognition 96, 215-231.

Tsakiris, M., Longo, M. R., and Haggard, P. (2010). Having a body versus moving your body: neural signatures of agency and bodyownership. Neuropsychologia 48, 2740-2749.

van den Bos, E., and Jeannerod, M. (2002). Sense of body and sense of action both contribute to self-recognition. Cognition 85, 177-187.
Verschure, P. F. M. J. (2011). Neuroscience, virtual reality and neurorehabilitation: brain repair as a validation of brain theory. Conf. Proc. IEEE Eng. Med. Biol. Soc. 2011, 2254-2257.

Vosgerau, G., and Synofzik, M. (2012). Weighting models and weighting factors. Conscious. Cogn. 21, 55-58.

Voss, M., Moore, J. W., Hauser, M., Gallinat, J., Heinz, A., and Haggard, P. (2010). Altered awareness of action in schizophrenia: a specific deficit in predicting action consequences. Brain 133, 3104-3112.

Wegner, D. M., and Sparrow, B. (2004). "Authorship processing," in The
Cognitive Neurosciences, eds M. Gazzaniga, R. B. Ivry, and G. R. Mangun (Cambridge, MA: MIT Press), 1201-1209.

Wegner, D. M., and Wheatley, T. (1999). Apparent mental causation. sources of the experience of will. Am. Psychol. 54, 480-492.

Yomogida, Y., Sugiura, M., Sassa, Y., Wakusawa, K., Sekiguchi, A., Fukushima, A., Takeuchi, H., Horie, K., Sato, S., and Kawashima, R. (2010). The neural basis of agency: an fMRI study. Neuroimage 50, 198-207.

Conflict of Interest Statement: The author declares that the research was conducted in the absence of any commercial or financial relationships that could be construed as a potential conflict of interest.

Received: 10 April 2012; accepted: 21 May 2012; published online: 01 June 2012

Citation: David N (2012) New frontiers in the neuroscience of the sense of agency. Front. Hum. Neurosci. 6:161. doi: 10.3389/fnhum.2012.00161

Copyright (c) 2012 David. This is an open-access article distributed under the terms of the Creative Commons Attribution Non Commercial License, which permits non-commercial use, distribution, and reproduction in other forums, provided the original authors and source are credited. 